University of New Orleans

ScholarWorks@UNO

4-1-1989

\title{
Maximum rate of change of the differential reflection phase shift with respect to the angle of incidence for light reflection at the surface of an absorbing medium
}

R. M.A. Azzam

University of New Orleans, razzam@uno.edu

A. M. El-Saba

Follow this and additional works at: https://scholarworks.uno.edu/ee_facpubs

Part of the Electrical and Electronics Commons

\section{Recommended Citation}

R. M. A. Azzam and A. M. El-Saba, "Maximum rate of change of the differential reflection phase shift with respect to the angle of incidence for light reflection at the surface of an absorbing medium," Appl. Opt. 28, 1365-1368 (1989)

This Article is brought to you for free and open access by the Department of Electrical Engineering at ScholarWorks@UNO. It has been accepted for inclusion in Electrical Engineering Faculty Publications by an authorized administrator of ScholarWorks@UNO. For more information, please contact scholarworks@uno.edu. 


\title{
Maximum rate of change of the differential reflection phase shift with respect to the angle of incidence for light reflection at the surface of an absorbing medium
}

\author{
R. M. A. Azzam and A. M. El-Saba
}

\begin{abstract}
The differential reflection phase shift, $\Delta=\delta_{p}-\delta_{s}$, associated with the external reflection of monochromatic light at the surface of an absorbing medium is a monotonically decreasing function of the angle of incidence $\phi$ which is determined by the complex dielectric function $\epsilon$. A new special angle of incidence, denoted by $\phi_{\Delta^{\prime} \max }$, is defined at which the slope $\Delta^{\prime}=\partial \Delta / \partial \phi$ of the $\Delta-\phi$ curve is maximum negative, $\Delta_{\max }^{\prime}$, and a transcendental equation is derived that determines this angle. $\phi_{\Delta^{\prime} \max }$ differs from the principal angle $\phi_{p}$ at which $\Delta=90^{\circ}$. As an example, $\phi_{\Delta^{\prime} \max }$ is calculated by numerical iteration for light reflection at the air-Si interface for photon energies $h \nu$ from 1.7 to $5.6 \mathrm{eV}$ in steps of $0.1 \mathrm{eV}$, and is plotted, along with the associated maximum slope $\Delta_{\max }^{\prime}$, vs wavelength $\lambda$. It is noted that $\phi_{\Delta^{\prime} \max }>\phi_{p}$ at every $\lambda$, a result that may hold in general. Also, for $4.5 \leq h \nu \leq$ $5.6 \mathrm{eV}, \phi_{\Delta^{\prime} \max }=90^{\circ}$, so that a maximum negative slope occurs at grazing incidence in this spectral range. Another interesting observation is that, when $|\epsilon| \gg 1$ (e.g., for metals in the IR), $\Delta^{\prime}\left(90^{\circ}\right)$ is a direct measure of the extinction coefficient $k=\operatorname{Im} \epsilon^{1 / 2}$.
\end{abstract}

\section{Introduction}

The ratio $\rho=r_{p} / r_{s}$ of the complex $p$ and $s$ Fresnel coefficients of light reflection at the planar interface between a transparent medium of incidence (usually air, $\left.\epsilon_{0}=1\right)$ and an absorbing medium of refraction $\left(\epsilon_{1}\right.$ complex) is given by ${ }^{1}$

$$
\rho=(A-B) /(A+B)
$$

where

$$
\begin{aligned}
& A=\sin ^{2} \phi, \\
& B=\cos \phi\left(\epsilon-\sin ^{2} \phi\right)^{1 / 2},
\end{aligned}
$$

$\phi$ is the angle of incidence, and

$$
\epsilon=\epsilon_{1} / \epsilon_{0}=\epsilon_{r}-j \epsilon_{i}
$$

is the complex relative dielectric constant. $\rho$, which is measured by ellipsometry, ${ }^{1}$ is often written in terms of its absolute value, $|\rho|=\tan \psi$, and angle $\Delta$ :

$$
\rho=|\rho| \exp (j \Delta) \text {. }
$$

When this work was done both authors were with University of New Orleans, Department of Electrical Engineering, Lakefront, New Orleans, Louisiana 70148; A. M. El-Saba is now with University of Alabama, Department of Electrical \& Computer Engineering, Huntsville, Alabama 35899.

Received 24 June 1988.

0003-6935/89/071365-04\$02.00/0.

(C) 1989 Optical Society of America.
In this paper we are interested in the behavior of the differential reflection phase shift, $\Delta=\delta_{p}-\delta_{s}$, as a function of $\phi$. It is known that, in the limiting case of a dielectric-dielectric interface $\left(\epsilon_{i}=0\right), \Delta=180^{\circ}$ for $\phi<$ $\phi_{B}$ and $\Delta=0$ for $\phi>\phi_{B}$, where $\phi_{B}=\tan ^{-1} \epsilon^{1 / 2}$ is the Brewster angle. Thus $\Delta$ vs $\phi$ is a step function, Fig. 1 (curve $a$ ) with an infinitely negative slope, $\partial \Delta / \partial \phi=$ $-\infty$, at $\phi_{B}$. However, in the presence of absorption in the second medium $\left(\epsilon_{i}>0\right)$, the discontinuous jump of $\Delta$ is replaced by a smooth transition, Fig. 1 (curve $b$ ), or gradual monotonic decrease of $\Delta$ from $180^{\circ}$ at $\phi=0$ (normal incidence) to 0 at $\phi=90^{\circ}$ (grazing incidence). In our discussion, we assume external reflection $(|\epsilon|>$ $1)$, the $\exp (j \omega t)$ time dependence of a time-harmonic monochromatic plane wave of light, and the associated Nebraska (Muller) conventions. ${ }^{2}$ More specifically, we are concerned with the slope or rate of change of $\Delta$ with respect to $\phi$,

$$
\Delta^{\prime}=\partial \Delta / \partial \phi,
$$

which can be measured directly by AIDER (angle of incidence derivative ellipsometry and reflectometry $\left.{ }^{3,4}\right)$. For an absorbing medium $\Delta^{\prime}$ is finite over the entire range of $\phi$ and is maximum negative at a certain angle of incidence that we denote by $\phi_{\Delta^{\prime} \max }$. This special angle, which, to our knowledge, has not been identified previously, differs from the pseudo-Brewster angle of minimum parallel reflectance and the principal angle at which $\Delta=90^{\circ} . \phi_{\Delta^{\prime} \max }$ is located by the condition that 


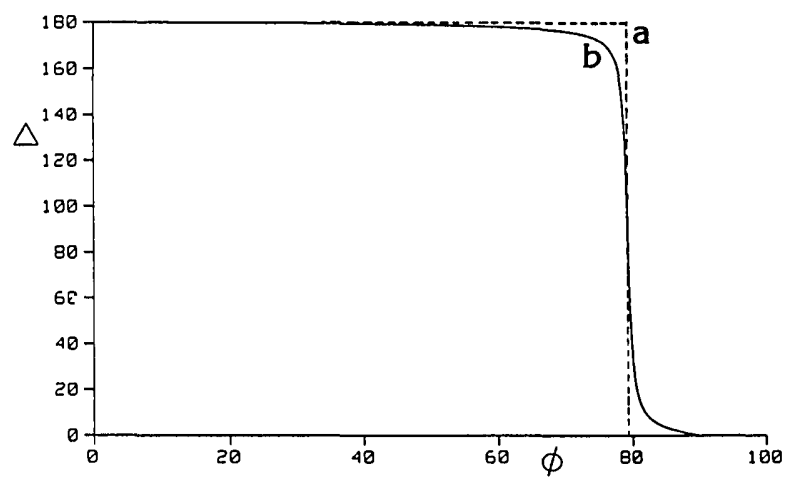

Fig. 1. Differential reflection phase shift $\Delta$ vs angle of incidence $\phi$ for a dielectric-dielectric interface (curve $a$ ) and dielectric-absorbing medium interface (curve $b$ ).

$$
\Delta^{\prime \prime}=\partial^{2} \Delta / \partial \phi^{2}=0 .
$$

This angle and the associated maximum negative slope are determined in this paper. Sections II and III give the required analytical expressions, and Sec. IV is an application to the reflection of monochromatic light at the vacuum or air-Si interface over the $1.7-5.6-\mathrm{eV}$ spectral range.

\section{Condition of Maximum Negative Slope}

If we take the derivative of the natural logarithm of both sides of Eq. (4), we obtain

$$
\rho^{\prime} / \rho=\left(|\rho|^{\prime} /|\rho|\right)+j \Delta^{\prime}
$$

where the prime superscript indicates the partial derivative with respect to $\phi$ throughout this paper. Consequently, from Eq. (7) we conveniently obtain

$$
\Delta^{\prime}=\operatorname{Im} F,
$$

where

$$
F=\rho^{\prime} / \rho \text {. }
$$

Taking the second partial derivative of Eq. (8) with respect to $\phi$, we get

$$
\Delta^{\prime \prime}=\operatorname{Im} G,
$$

where

$$
G=F^{\prime}=\left(\rho \rho^{\prime \prime}-\rho^{2}\right) / \rho^{2} .
$$

With $\rho$ expressed in the form of Eq. (1), we find that

$$
\begin{aligned}
& F=\left(A^{\prime} B-A B^{\prime}\right) /\left(A^{2}-B^{2}\right), \\
& G=\frac{\left(A^{2}-B^{2}\right)\left(A^{\prime \prime} B-A B^{\prime \prime}\right)-2\left(A^{\prime} B-A B^{\prime}\right)\left(A A^{\prime}-B B^{\prime}\right)}{\left(A^{2}-B^{2}\right)^{2}} .
\end{aligned}
$$

For completeness, the derivatives $A^{\prime}, B^{\prime}, A^{\prime \prime}$, and $B^{\prime \prime}$, as obtained from Eqs. (2), are listed below:

$$
\begin{aligned}
& A^{\prime}=\sin 2 \phi, \\
& B^{\prime}=-\sin \phi\left(\epsilon-\sin ^{2} \phi\right)^{-1 / 2}(\epsilon+\cos 2 \phi), \\
& A^{\prime \prime}=2 \cos 2 \phi \\
& B^{\prime \prime}=-\cos \phi\left(\epsilon-\sin ^{2} \phi\right)^{-3 / 2}\left[\epsilon^{2}+\left(1-6 \sin ^{2} \phi\right) \epsilon+4 \sin ^{2} \phi\right] .
\end{aligned}
$$

The angle of incidence of maximum negative slope is determined by $\Delta^{\prime \prime}=0$ or

$$
\operatorname{Im}[G(\phi)]=0 .
$$

Equation (15), where $G$ is given by Eqs. (13), (2), and (14), is solved by numerical iteration for any given complex $\epsilon$. The associated $\Delta_{\max }^{\prime}$ is calculated subsequently using Eqs. (8), (12), (2), and (14).

\section{III. $\Delta^{\prime}$ and $\Delta^{\prime \prime}$ at Normal and Grazing Incidence}

It is instructive to consider the limiting values of the derivatives $\Delta^{\prime}$ and $\Delta^{\prime \prime}$ at $\phi=0$ and $\phi=90^{\circ}$. At normal incidence, $\phi=0$, Eqs. (12) and (14) show that

$$
F(0)=0 \text {, }
$$

hence

$$
\Delta^{\prime}(0)=0
$$

from Eq. (8). This result is not new and is consistent with the known stationary property of normal incidence reflection. ${ }^{5}$ Likewise, from Eqs. (13) and (14) we obtain

$$
G(0)=-2 \epsilon^{-1 / 2},
$$

hence

$$
\Delta^{\prime \prime}(0)=-2 \operatorname{Im} \epsilon^{-1 / 2}
$$

from Eq. (10).

At grazing incidence, $\phi=90^{\circ}$, we get (from the same equations of Sec. II)

$$
\begin{aligned}
F\left(90^{\circ}\right) & =2(\epsilon-1)^{1 / 2}, \\
\Delta^{\prime}\left(90^{\circ}\right) & =2 \operatorname{Im}(\epsilon-1)^{1 / 2}, \\
G\left(90^{\circ}\right) & =0, \\
\Delta^{\prime \prime}\left(90^{\circ}\right) & =0 .
\end{aligned}
$$

To summarize, at $\phi=0, \Delta^{\prime}=0$ and $\Delta^{\prime \prime} \neq 0$; conversely, at $\phi=90^{\circ}, \Delta^{\prime} \neq 0$ and $\Delta^{\prime \prime}=0$. The nonzero derivatives are given by Eqs. (19) and (21).

Equation (23) indicates that the slope $\Delta^{\prime}$ is at an extremum (a maximum or a minimum), given by Eq. (21), at $\phi=90^{\circ}$. In fact, depending on complex $\epsilon, \phi=$ $90^{\circ}$ may be the only solution of Eq. (15), so that the maximum negative slope occurs at grazing incidence:

$$
\left.\Delta_{\max }^{\prime}\right|_{\phi=90^{\circ}}=2 \operatorname{Im}(\epsilon-1)^{1 / 2} .
$$

If $|\epsilon| \gg 1$, e.g., for metals in the IR, Eq. (21a) can be approximated by

$$
\left.\Delta_{\max }^{\prime}\right|_{\phi=90^{\circ}}=2 \operatorname{Im} \epsilon^{1 / 2}=-2 k,
$$

where the complex refractive index is

$$
N=\epsilon^{1 / 2}=n-j k .
$$

Equation (24) is interesting because it shows that the limiting slope of the $\Delta$ vs $\phi$ curve as grazing incidence is approached is a direct measure of the extinction coefficient $k$. 

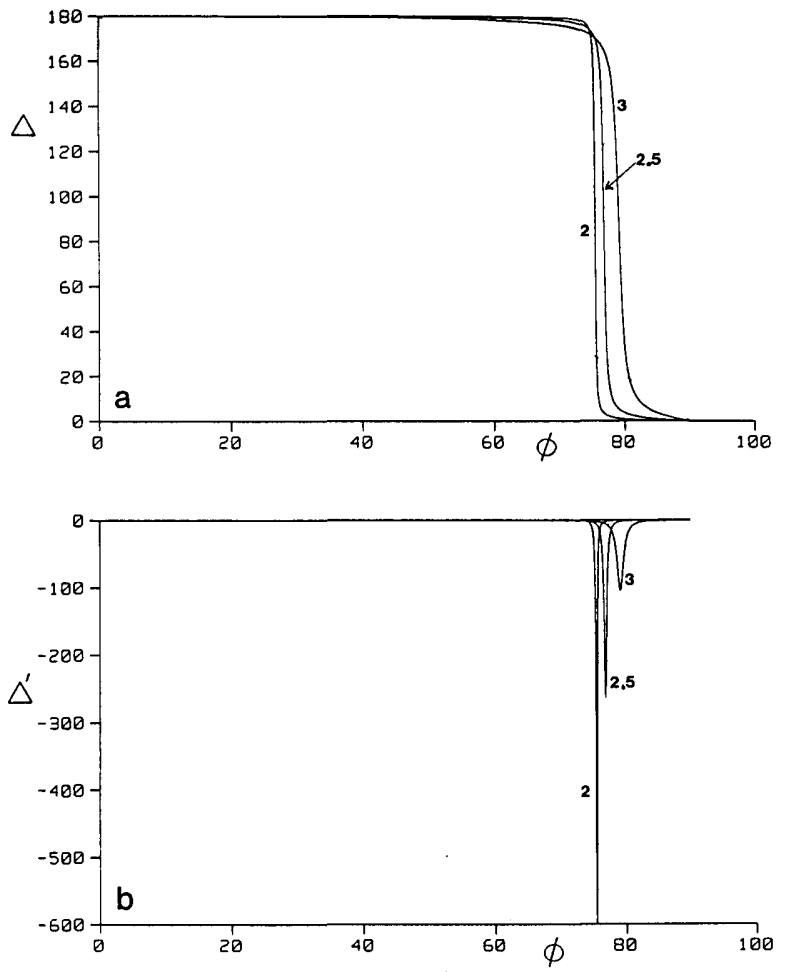

Fig. 2. (a) $\Delta$ vs $\phi$ curves for the air-Si interface at photon energies $h \nu=2.0,2.5$, and $3 \mathrm{eV}$ as marked by each curve. (b) Derivative $\Delta^{\prime}=$ $\partial \Delta / \partial \phi$ of the curves in (a) plotted vs $\phi$.


Fig. 3. Same as Fig. 2 except that here $h \nu=3.5$ and $4 \mathrm{eV}$.
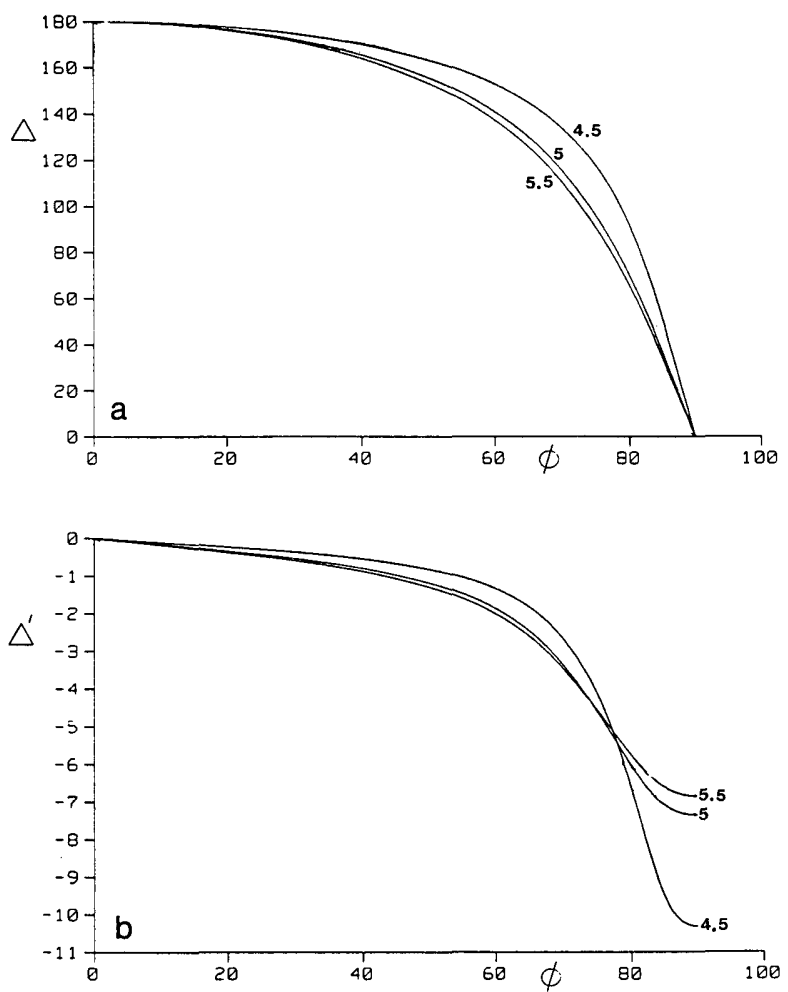

Fig. 4. Same as Fig. 2 except that here $h \nu=4.5,5.0$, and 5.5. eV.

\section{Light Reflection at the Vacuum or Air-Si Interface: An Example}

Values of the complex dielectric function of $\mathrm{Si}, \epsilon(h \nu)$, at different photon energies $h \nu$ are taken from Aspnes and Studna ${ }^{6}$ and are included in Table I for completeness.

Figure 2(a) shows $\Delta$ vs $\phi$ for $h \nu=2.0,2.5$, and $3.0 \mathrm{eV}$, and Fig. 2(b) gives the associated derivative curves of $\Delta^{\prime}$ vs $\phi$. In this range of photon energies, $\epsilon_{i}$ is small compared with $\epsilon_{r}\left(\epsilon_{i} / \epsilon_{r} \lesssim 0.1\right)$ and the $\Delta-\phi$ curve exhibits a steep but smooth transition in the neighborhood of the principal angle $\phi_{p}$ of $\Delta=90^{\circ}$ (or the pseudoBrewster angle of minimum parallel reflectance which differs from $\phi_{p}$ by $\left.<0.003^{\circ}\right)$. The $\Delta^{\prime}-\phi$ curve, therefore, takes the shape of a narrow negative pulse whose peak is positioned at the point of inflection of the $\Delta-\phi$ curve and of magnitude equal to $\Delta_{\text {max }}^{\prime}$.

Figures 3(a) and (b) show $\Delta$ and $\Delta^{\prime}$ vs $\phi$ for $h \nu=3.5$ and $4.0 \mathrm{eV}$. In this case, $\epsilon_{i}>\epsilon_{r}$ and $\Delta$ decreases more gradually as $\phi$ increases. The height of the negative peak of the $\Delta^{\prime}-\phi$ curve is significantly reduced and the slope at grazing incidence $\Delta^{\prime}\left(90^{\circ}\right)$ is significantly increased when compared with these characteristics for $h \nu=2.0,2.5$, and $3.0 \mathrm{eV}$ shown in Fig. 2(b).

Figures 4(a) and (b) give the corresponding results for $h \nu=4.5,5.0$, and $5.5 \mathrm{eV}$. In this case, the point of inflection of the $\Delta-\phi$ curve disappears and the maximum negative slope is at grazing incidence. The $\Delta^{\prime}-\phi$ curve has a single extremum $\left(\Delta^{\prime \prime}=0\right)$, a minimum, at $\phi$ $=90^{\circ}$.

Table I lists the angle of incidence $\phi_{\Delta^{\prime} \max }$ of maximum negative slope, the maximum negative slope 
Table I. Maximum Slope of the $\Delta-v s-\phi$ Curve, $\Delta_{\max }^{\prime}$, and the Angle of Incidence at Which it Occurs, $\phi_{\Delta^{\prime} \max }$, for Si at Different Photon Energies ${ }^{a} h \nu$

\begin{tabular}{cccccccc}
\hline$h \nu(\mathrm{eV})$ & $\lambda(\mu \mathrm{m})$ & \multicolumn{1}{c}{$\epsilon_{r}$} & $\epsilon_{i}$ & $\phi_{\Delta^{\prime} \max }\left({ }^{\circ}\right)$ & $\phi_{p}\left({ }^{\circ}\right)$ & $\phi_{\Delta^{\prime} \max }-\phi_{p}$ & $\Delta_{\max }^{\prime}$ \\
\hline 2.0 & 0.620 & 15.254 & 0.172 & 75.6391 & 75.6390 & 0.0002 & -738.2270 \\
2.5 & 0.496 & 18.661 & 0.630 & 76.9714 & 76.9699 & 0.0015 & -269.8299 \\
3.0 & 0.413 & 27.197 & 2.807 & 79.1862 & 79.1742 & 0.0120 & -105.5199 \\
3.5 & 0.354 & 22.394 & 33.818 & 82.1509 & 81.1242 & 1.0267 & -14.7124 \\
4.0 & 0.310 & 12.240 & 35.947 & 82.6085 & 80.8616 & 1.7469 & -12.0930 \\
4.5 & 0.275 & -19.815 & 24.919 & 90.0000 & 80.1862 & 9.8138 & -10.3232 \\
5.0 & 0.248 & -10.240 & 11.195 & 90.0000 & 76.2770 & 13.723 & -7.3631 \\
5.5 & 0.225 & -9.106 & 8.846 & 90.0000 & 75.1957 & 14.804 & -6.8610 \\
\hline
\end{tabular}

${ }^{a} \lambda$ is the corresponding wavelength of light, $\epsilon_{r}, \epsilon_{i}$ are the real and imaginary parts of the complex dielectric function of Si from Ref. 6, and $\phi_{p}$ is the principal angle at which $\Delta=90^{\circ}$.

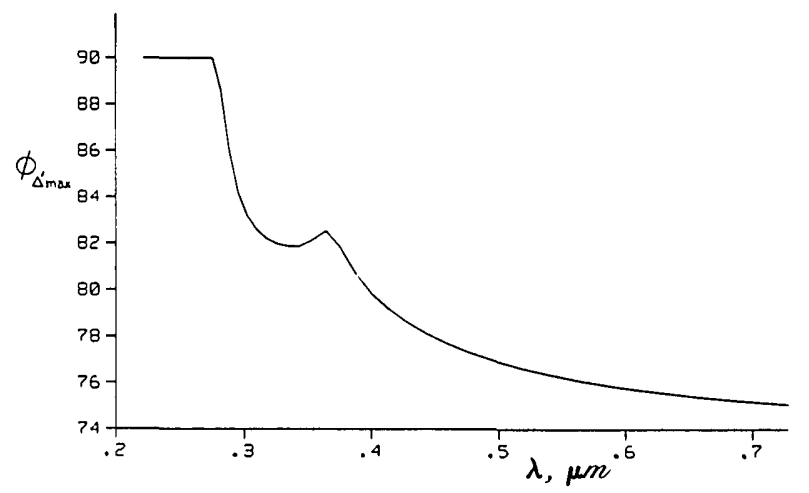

Fig. 5. Angle of incidence, $\phi_{\Delta^{\prime} \max }$, of the maximum negative rate of change of $\Delta$ with respect to $\phi$ plotted vs wavelength $\lambda$ for light reflection at the air-Si interface.



Fig. 6. Maximum negative rate of change of $\Delta$ with respect to $\phi$, $\Delta_{\max }^{\prime}$, plotted vs wavelength $\lambda$ for the air-Si interface.

$\Delta_{\max }^{\prime}$, for Si at $h \nu$ from 2.0 to $5.5 \mathrm{eV}$ in steps of $0.5 \mathrm{eV}$, that correspond to the graphic results of Figs. (2)-(4). Also listed are the principal angle $\phi_{p}$ and the difference $\phi_{\Delta^{\prime} \max }-\phi_{p}$. The latter is positive at every $h \nu$ so that the angle of maximum $\Delta-\phi$ slope is always above the principal angle of $\Delta=90^{\circ}$.

Finally, Fig. 5 shows $\phi_{\Delta^{\prime} \max }$ vs $\lambda$ and Fig. 6 shows $\Delta_{\max }^{\prime}$ vs $\lambda$ for Si for wavelengths $\lambda$ between 0.221 and 0.729 $\mu \mathrm{m}$. This corresponds to the range of $h \nu$ from 1.7 to 5.6 $\mathrm{eV}$ with the calculations made at each step of $0.1 \mathrm{eV}$.

\section{Summary}

Several special angles associated with the external reflection of light at the surface of an absorbing medium are well known. ${ }^{7}$ They include the pseudo-Brewster angle of minimum parallel reflectance $\left|r_{p}\right|$, the second Brewster angle of minimum reflectance ratio $\left|r_{p} / r_{s}\right|$, and the principal angle $\phi_{p}$ at which the differential reflection phase shift $\Delta=90^{\circ}$. In this paper we identify a fourth significant angle, $\phi_{\Delta^{\prime} \text { max }}$, at which the rate of change $\Delta^{\prime}=\partial \Delta / \partial \phi$ (which is measurable by AIDER $^{3,4}$ ) is maximum negative. The equation that governs this angle is derived and solved, by numerical iteration, for light reflection at the air-Si interface over a wide spectral range. Results are presented in Figs. 2-6 and in Table I. $\Delta_{\max }^{\prime}>\phi_{p}$ for Si at every wavelength, a result that may hold in general. Also, at short wavelengths, $\phi_{\Delta^{\prime} \max }=90^{\circ}$ and the maximum slope of the $\Delta-\phi$ curve occurs at grazing incidence. It is also noted that when $|\epsilon| \gg 1$ (e.g., for metals in the IR), $\Delta^{\prime}\left(90^{\circ}\right)$ gives a direct measure of the extinction coefficient $k=\operatorname{Im} \epsilon^{1 / 2}$.

\section{References}

1. R. M. A. Azzam and N. M. Bashara, Ellipsometry and Polarized Light (North-Holland, Amsterdam, 1977).

2. R. H. Muller, "Definitions and Conventions in Ellipsometry," Surf. Sci. 16, 14 (1969).

3. R. M. A. Azzam, "AIDER: Angle-of Incidence Derivative Ellipsometry and Reflectometry," Opt. Commun. 16, 153 (1976).

4. V. M. Bermudez, "AIDER (Angle-of Incidence Derivative Ellipsometry and Reflectometry): Implementation and Application," Surf. Sci. 94, 29 (1980).

5. R. M. A. Azzam, "Stationary Property of Normal-Incidence Reflection from Isotropic Surfaces," J. Opt. Soc. Am. 72, 1187 (1982).

6. D. E. Aspnes and A. A. Studna, "Dielectric Functions and Optical Parameters of $\mathrm{Si}, \mathrm{Ge}, \mathrm{GaP}, \mathrm{GaAs}, \mathrm{GaSb}$, InP, InAs, and InSb from 1.5 to $6.0 \mathrm{eV}$," Phys. Rev. B 27, 985 (1983).

7. See, for example, J. M. Bennett and H. E. Bennett, "Polarization," in Handbook of Optics, W. G. Driscoll and W. Vaughan, Eds. (McGraw-Hill, New York, 1978). 